\title{
A CASE OF ACUTE PYREXIAL PUSTULAR DERMATITIS.
}

\author{
BY LESLIE PHILLIPS, M.D.,
}

Surgeon to the Birmingham Hospital for Skin and Urinary Diseases.

As a contribution to the at present obscure subject of generalized pustular eruptions apparently depending on a systemic condition or infection rather than on a local causation, the record of the following case is not without interest :-

Mary K., married, aged 27. Was first seen on July 17, 1897. She was obviously ill, and was admitted as an in-patient. The skin presented a remarkable and striking eruption. This had begun on July 9, although for a week previously she had been complaining. The rash first appeared on the backs of the knuckles and of the wrists, and then on the anterior surface of the legs. The eruption got steadily worse and the constitutional disturbance more pronounced. When seen the rash affected the back of the arms, forearms, and hands, and the posterior aspect of the trunk; there was practically none on the anterior aspect of the trunk. On the lower limbs both surfaces were equally and much affected, especially towards the lower part. The general distribution of the eruption is shown in Fig. 1, from a photographic memorandum taken on the tenth day of the efflorescence.

The element of the rash was an inflamed, irregular-shaped or roundish patch, seated on which are many primary pustules, each nearly as large as a pea (Fig. 2). Each of the patches looked herpetic, and except that the lesions were pustular from the beginning a description of Zoster would nearly describe the appearance of a patch of the eruption. There are groups of large discrete pustules seated on a red, painful, tender, and swollen base of skin.

The containing-membrane of the lesions was strong, and the pustules were consequently resisting and did not break either spontaneously or with moderate handling; neither did crusts form, nor did eczematous excoriations follow the lesions. Involution took place by drying or absorption, beginning in the centre of the patch and leaving the centre of a fawn or yellowish colour, while perfect pustules still persisted at the periphery. Pustules disappeared from some of the patches, while on others they still remained present, depending upon the age of the patch. This was the only approach to "croppiness" about the eruption, for, on placing the patient in bed, the evolution soon ceased, and disappearance became gradual, continuous, and uninterrupted. On the twentieth day of the eruption the patient was, with the exception of debility, well. 
Some pustules became confluent before they dried. I did not note that fresh pustules appeared to any extent on the periphery while the involution of the central ones was taking place, although in many patches the centre became quite dried and fawn-coloured, while a circle of pustules still remained at the periphery.

There was considerable constitutional disturbance, the patient being, and looking, acutely and gravely ill. The temperature was pyrexial, as shown in the chart (Fig. 3).

The tongue was swollen, very coated with creamy-white fur, and the odour perceived on approaching the bed was like that of typhoid fever. The pulse rate was not very rapid, never exceeding 90 (her pulse, probably, was naturally slow,

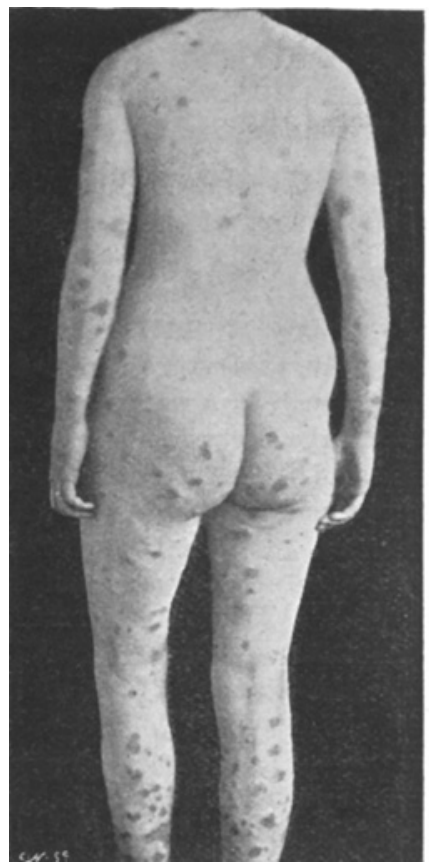

FIG, 1.

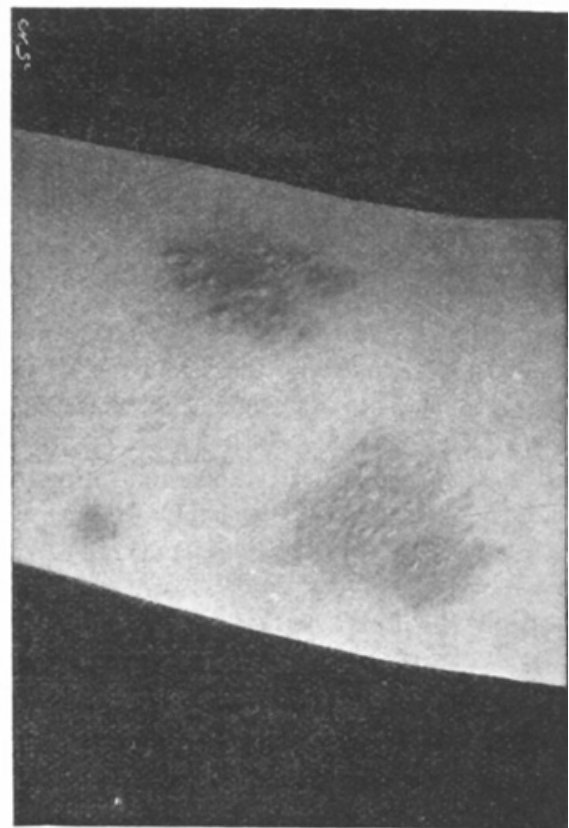

FIG. 2.

about 60). The subsidence of the general pyrexia coincided with the disappearance of the local lesions, not, as is the case in Hebra-Kaposi's description of Impetigo herpetiformis, with its appearance. As the patches cleared they left a reddish, desquamating stain, indicating the position of the lesions. No antecedent cause of the disease could be elicited, but there is reason to believe the patient's domestic life was unhappy. She was not pregnant. A culture was unfortunately accidentally contaminated.

In casting about for a name for this condition, the one which seemed naturally suggested was Impetigo herpetiformis, had not that been already hypothecated by Hebra and Kaposi to the disease they 
have described, and which they claim is a distinct and well-marked entity, alternatively named by Auspitz,-Herpes pyæmicus. Although in some respects analogous, especially in being pyrexial, the present case does not, however, correspond with the description given by the masters of the Vienna School, either in origin, course, or termination. In Kaposi's description, the herpetiform pustules are the size of pins'-heads, the patch also begins small, it crusts, and there is a

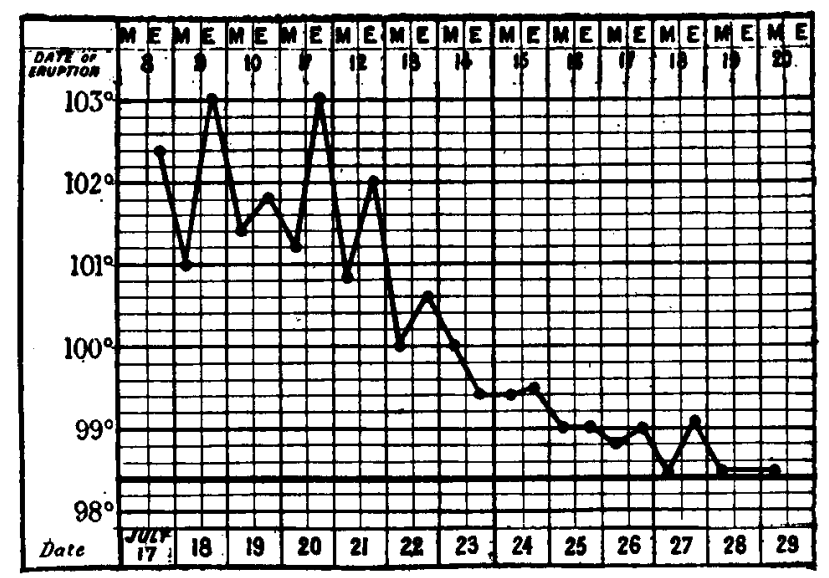

FIG. 3.

gradual augmentation of the first crust by a similar process going on at the circumference by successive generations of lesions like the first. In three or four months the whole skin is invaded, and is crusted and excoriated. There is a remission of the fever with the appearance of each crop of efflorescence.

As the original description of Impetigo herpetiformis has proved too absolute, both in regard to the supposed invariable fatal prognosis and to its being confined to pregnant women, so, as observations multiply, it may be found (as Crocker seems to suggest) that the magistral description is too restrictive and gives too "narrow a conception of the disease."

The present case, moreover, does not correspond with that described, in 1889, by Hallopeau, under the name "Nouvelle forme de dermatite pustuleuse en foyer, à progression excentrique." Although in certain local respects resembling it, such resemblances 
rather appear to belong to a group of pustular dermatoses, owning a pyæmic or systemic pyococcic infection. The characters of such a group being: grouped pustules arranged in circular or irregular patches, commencing to heal centrally while still persisting or spreading at the periphery. In Hallopeau's case the pustules began as vesico-pustules, were miliary as in Impetigo herpetiformis, and led, as in that disease, to thick crusting; while intense pruritus was noted, the general health remained good, and the disease was chronic. The case now described was acute, septicæmic, pyrexial, attended with severe constitutional disturbance, and ran a distinct course towards recovery. It seems to confirm the correctness of the tendency of French dermatological opinion, that such a pyodermia is to be regarded as a member of a group rather than as a specific entity. It may be that the essential is a pyococcic intoxication, and it seems likely that the pathogenic functions of pyogenic cocci acting systemically may result in manifestations too diverse for absolutely definite particularisation, while at the same time producing consequences of a pyæmic order, which may conveniently, for the present at any rate, be relegated to a group.

Hallopeau probably approximates the correct pathology when he attributes these cases to "a form of chronic (?) pyogenic infection, limited to the integuments."

No small amount of dogmatism is required to claim that the size of pustules, chronicity or acuteness, small details of distribution, or variations in course, the absence or presence of pruritus and similar modifications, constitute essential features of a disease which is of the nature of a pyococcic invasion. It is easier to understand that divergences from the type may be caused by varying degrees of general or local resistance or immunity, and by those differences of soil which constitute the personal or individual element in the equation of any disease. 